\title{
Edges Enhancement of Medical Color Images Using Add Images
}

\author{
Dr. Ziad M. Abood \\ Mustansiriyah University/ College of Education
}

\begin{abstract}
It is important; medical images to be clear, because it will be built upon diagnosis of the patient's condition, and the degree of illness, and its appropriate treatment.

The aim of present study is clarify the edges of color medical images by increasing the thickness to get rid of soft edges and some areas that do not appear when the edge is selected.

It Method was used to add images by Matlab2012a program, finding the edge of medical images, and then add the resulting images to the original images, therefore, be fully match their home being with the same characteristics of size, features and more importantly, matching sites pixellike.

This proposed method can the application of all types of medical images (MRI, CT, CAT, PAT,..) color and gray, with any size and any member of human's body.

Results gave high accuracy with the output images, if clarity and showed an increase in the thickness of the edges of the output images.

Keywords: Medical Image Enhancement, Edges Detection, Add Images, Matlab.
\end{abstract}

\section{Introduction}

Medical image enhancement technologies have attracted much attention since advanced medical equipment's were put into use in the medical field. Enhanced medical images are desired by a surgeon to assist diagnosis and interpretation because medical image qualities are often deteriorated by noise and other data acquisition devices, illumination conditions, etc. Also targets of medical image enhancement are mainly to solve problems of low contrast and the high level noise of a medical image. Medical image enhancement technologies have attracted many studies [1].The basic color space (RGB) conversion to other color spaces is important mainly concentrated on the edges enhancement of medical color image.

\section{- Previous studies:}

Muna F. et al [2],thispaper proposes a novel method for enhancing and sharpening medical color digitalimages. Low contrast and poor quality are main problems in the production of medicalimages. By using the wavelet transforms and Haar transform followed by using Sobel and Laplacian operator to obtain the sharpened image. First, a medical image wasdecomposed with wavelet transform. Secondly, all high-frequency sub-images were decomposed with Haar transform. Thirdly, noise in the frequency field was reduced by thesoftthreshold method. Fourthly, high-frequency coefficients were enhanced by differentweight values in different sub-images. Then, the enhanced image was obtained through theinverse wavelet transform and inverse Haar transform. Lastly, the filters are applied tosharpen the image; the resulting image is then subtracted from the original image.Experiments showed that this method can not only enhance an image's details but can alsopreserve its edge features effectively.

Hajj et al 1998 [3],a general framework is presented in this paper for edge detection and enhancement of medical images. The method is based on multiscale analysis using filter banks, and it is adaptive to a large number of features. Initially, an optimal one-scale filter is designed for the required detection. This one-scale filter is further extended to a set of multiscale filters, which in turn are used in designing the filter bank that would provide the desired multiscale responses. Subsequently, the scale space information is optimally combined in a maximumposteriori (MAP) classifier, whose design depends on the desired feature and the resulting filter bank. The method is robust to noisy conditions which are common to medical images in angiography, echocardiography, blood vessels, and others based on ultrasonic imaging, X-ray, and tomography. Gudmundsson et al 1998 [4],an algorithm is developed that detects well-localized, unfragmented, thin edges in medical images based on optimization of edge configurations using a genetic algorithm (GA). Several enhancements were added to improve the performance of the algorithm over a traditional GA. The edge map is split into connected subregions to reduce the solution space and simplify the problem. The edge-map is then optimized in parallel using incorporated genetic operators that perform transforms on edge structures. Adaptation is used to control operator probabilities based on their participation. The GA was compared to the simulated annealing (SA) approach using ideal and actual medical images from different modalities including magnetic resonance imaging (MRI), computed tomography (CT), and ultrasound. Quantitative comparisons were provided based on the Pratt figure of merit and on the cost-function minimization. The detected edges were thin, continuous, and well localized. Most of the basic edge features were detected;results for different medical 
image modalities are promising and encourage further investigation to improve the accuracy and experiment with different cost functions and genetic operators.

Tarek et al 1998 [5],one of the most common degradations in medical images is their poor contrast quality. This suggests the use of contrast enhancement methods as an attempt to modify the intensity distribution of the image. In this paper, a new edge detected morphological filter is proposed to sharpen digital medical images. This is done by detecting the positions of the edges and then applying a class of morphological filtering. Motivated by the success of threshold decomposition, gradient based operators are used to detect the locations of the edges. A morphological filter is used to sharpen these detected edges. Experimental results demonstrate that the detected edge deblurring filter improved the visibility and perceptibility of various embedded structures in digital medical images. Moreover, the performance of the proposed filter is superior to that of other sharpener-type filters.

Zhao et al 1998 [6], medical images edge detection is an important work for object recognition of the human organs and it is an important pre-processing step in medical image segmentation and 3D reconstruction. Conventionally, edge is detected ac-cording to some early brought forward algorithms such as gradient-based algorithm and template-based algorithm, but they are not so good for noise medical image edge detection. In this paper, basic mathematical morphological theory and operations are introduced at first, and then a novel mathematical morphological edge detection algorithm is proposed to detect theedge of lungs CTimage withsaltand-peppernoise. Theexperimentalresultsshowthat the proposedalgorithm is moreefficientformedicalimagedenoising and edge detectionthantheusuallyused template-based edgedetection algorithms andgeneral morphologicaledgedetectionalgorithms.

Lakshmi et al 1998 [7], medical Image Processing and its applications in Computer Assisted Diagnoses (CAD) and therapy (e.g. Computer Assisted Surgery-CAS) are of increasing importance in modern medicine. The most common degradations in medical images are their poor contrast quality. Medical Image Enhancement is the art of examining images for identifying objects and judging their significance. The proposed paper uses the concept of Genetic Algorithm which was proved to be the most powerful unbiased Optimization techniques for sampling a large solution space. Because of unbiased stochastic sampling, they can be quickly adapted in medical image processing. They can be applied for the medical image enhancement, segmentation, feature extraction, classification and image generation. In this paper, we deal with medical image enhancement using Genetic Algorithm (GA) and the Morphological filter to sharpen the detected edges thus improving the contrast of the image.

In this paper, was proposed method of image enhancement based on edge detection with adding image. The structure of the paper is arranged as follows:section 1 included the introduction and section 2included the background of theoretical.The samples images and proposed scheme of Algorithmin section 3. Section 4 included the results. Conclusions and future works are shown in section 5.

\section{Background}

\subsection{Image Enhancement}

Image enhancement is a process principallyfocuses on processing an image in such a way thatthe processed image is more suitable than theoriginal one for the specific application. The word"specific" has significance. It gives a clue that theresults of such an operation are highly applicationdependent. In other words, an image enhancementtechnique that works well for X-ray topographicimages may not work well for MR images.The technique falls in two categories on thebasis of the domain they are applied on. These arethe frequency and spatial domains. The frequency domainmethods work with the Fourier Transformsof the image. The term spatial domain refers to thewhole of pixels of which an image is composed of.Spatial domain methods are procedures that operatedirectly on the pixels. The process can be expressedas: [8]

$$
g(x, y)=T[f(x, y)]
$$

Where $f(x, y)$ is the input image, $g(x, y)$ is theprocessed image, and $T$ is an operator on $f$ definedover some neighborhood of $(\mathrm{x}, \mathrm{y})$. Number of enhancement techniques exists in the spatial domain.Among these are histogram processing, enhancementusing arithmetic, and logical operations and filters.

\subsection{Medical Images}

Medical images are a special kind of images. These images are used for the diagnostics of diseasesin the patients $[9,10]$. A number of modalities existfor obtaining these images. Among popular ones are Computed Topographic Imaging (CT), MagneticResonance Imaging (MRI), etc. Our focus here willbe on the image obtained through MagneticResonance Imaging (MRI).

Biologic tissues are comparatively transparent tox-rays and opaque to radiation with intermediatewavelengths when proceeding from the shorter to thelonger wavelengths of the electromagnetic spectrum. This is true for ultraviolet, visible, and, to someextent, infrared light and microwaves. However,there is a window in tissue absorption through withradio waves can be used to probe deep inside thehuman body. The 
benefits derived from low-energyradiation and unprecedented level of informationavailable from nuclear signals combined to makeimaging by magnetic resonance a valuablebiomedical imaging modality. [11]

Magnetic Resonance Cholangiography (MRC) isan imaging method using a magnetic resonanceimaging (MRI) scanner. Because MRC can acquirethe pancreatic duct with a high MR signal, it hasbeen widely used for diagnosing diseases of thepancreatic duct, such as calculi and pancreatitis.However, there are some limitations for use of MRC:first MRC images often involve other tissues (e.g.,fat, stomach) that have a high MR signal becauseMRC imaging method gives a high MR signal forwater. Therefore, when we generate 2D projectedimages by means of maximum intensity projection(MIP), volume rendering (VR), or others, thosetissues with high MR signal will overlap with thepancreatic duct and secondly, If we use a lowtestMRI or a thick slice of imaging parameter to reducethe imaging cost or to be faster the imaging time,some parts of the pancreatic duct will disappearbecause of a partial volume effect (PVE). Such lacksmay impede the physicians' observation, and mightlead to a miss-diagnosis that is, the problem is thatthe MR signal of the pancreatic duct is lower than orequals MR signals of the other tissues. Therefore, uses of image enhancement techniques willcontribute to overcoming these limitations. [12]

\subsection{Edge Detection}

Edge detection refers to the process of identifying and locating sharp discontinuities in an image. The discontinuities are abrupt changes in pixel intensity which characterize boundaries of objects in a scene. Classical methods of edge detection involve convolving the image with an operator (a 2-D filter), which is constructed to be sensitive to large gradients in the image while returning values of zero in uniform regions.

Existing methods use aNxN matrix known as kernel to conclude decisions of a point being part of a potential edge. In general and most cases, $\mathrm{N}=3$ where the number of cells in the matrix is 9. Matrix operations are thus performance costly. Also with the increase in the value of $\mathrm{N}$ to 4 or 5 or more, the calculations become more complex and duration of first pass results keep increasing. Many existing methods propose to use noise reduction filter as a pre-requisite for edge detection, thus increasing an additional pass in image processing. [13] Edge detection is generally todefine edges of the object inside the image. Edgescan be found when the difference between luminanceintensity from one point to the other appears. Practically, the more difference of light luminance,the edges are easier to define. In contrast, the lesserthe difference the harder the edges can be define.Edge detection evaluates the brightness of each area with difference luminance.[14]

The filtering technique in this research wasperformed by using Canny,Laplacian and Sobel technique,due to its evaluation capacity which filters the imageconstantly and spontaneously. This technique facesseveral limitations i.e., adding thick layers to theedges due to its slope from the result of firstderivative computation at different grayscale level;from highest to lowest or conversely opposition. Inaddition, the result of the first derivative leads to theincorrect grayscale level from the original. Bycomputing the second derivative we obtain Laplacianmethod which was proven to have a better filteringresult than the first method. This method separatesthe thin layers of the area and determines thedifferences of each pixel accurately. Therefore, the benefits of the Laplacianmethod completely outweigh those of gradient edge detection theequation is as follows: [15]

$$
\nabla^{2} \mathrm{f}(\mathrm{x}, \mathrm{y})=\mathrm{f}(\mathrm{x}+1, \mathrm{y})+\mathrm{f}(\mathrm{x}-1, \mathrm{y})+\mathrm{f}(\mathrm{x}, \mathrm{y}+1)+\mathrm{f}(\mathrm{x}, \mathrm{y}-1)-4 \mathrm{f}(\mathrm{x}, \mathrm{y}) \ldots(1)
$$

Edge detection with Laplacian has limitations aswell. The kernel is another important circumstance todetermine the most suitable kernel for the pixelsfiltered. Sobel is considered a well replacement ofLaplacian filter. According to the Sobelcharacteristics, the total factor of the filter equals tozero which in certain cases it can reduce theoverflow problematic [15]. In digital images $f(x, y)$,Sobel operator, $S(x, y)$ in the convolution procedureand image were set to as the coefficients below:

$$
\begin{aligned}
\mathrm{A}= & {[\mathrm{f}(\mathrm{x}-1, \mathrm{y}+1)+2 \mathrm{f}(\mathrm{x}-1, \mathrm{y})+\mathrm{f}(\mathrm{x}-1, \mathrm{y}-1)] } \\
& -[\mathrm{f}(\mathrm{x}-1, \mathrm{y}+1)+2 \mathrm{f}(\mathrm{x}+1, \mathrm{y})+\mathrm{f}(\mathrm{x}+1, \mathrm{y}-1)] \ldots(2 \mathrm{a}) \\
\mathrm{B}= & {[\mathrm{f}(\mathrm{x}-1, \mathrm{y}-1)+2 \mathrm{f}(\mathrm{x}, \mathrm{y}-1)+\mathrm{f}(\mathrm{x}, \mathrm{y}-1)] } \\
& +[\mathrm{f}(\mathrm{x}+1, \mathrm{y}-1)+2 \mathrm{f}(\mathrm{x}, \mathrm{y}+1)+\mathrm{f}(\mathrm{x}+1, \mathrm{y}+1)] \ldots(2 \mathrm{~b}) \\
\mathrm{S}_{\mathrm{m}}(\mathrm{x}, \mathrm{y})= & \left(\mathrm{A}^{2}+\mathrm{B}^{2}\right)^{1 / 2} \ldots(3)
\end{aligned}
$$

A: The elements in the first and third column,

B: The elements in the first and third row.

After estimating above equation, we obtain:

$\mathrm{S}_{\mathrm{m}}(\mathrm{x}, \mathrm{y}) \approx \mathrm{A}+\mathrm{B}$ 
Once we have our luminance map, we can use the Sobel operator to detect edges.

The Sobel operator calculates the directional change of the image intensity at each point, giving the direction of the largest possible increase from light to dark and the rate of change in that direction, using uses a pair of $3 \times 3$ convolution masks, one estimating the directional change in the $\mathrm{x}$-direction and the other estimating the directional change in the y-direction. This is the convolution mask: [16]

$\mathbf{G}_{y}=\left[\begin{array}{ccc}-1 & -2 & -1 \\ 0 & 0 & 0 \\ +1 & +2 & +1\end{array}\right] * \mathbf{A}$ and $\mathbf{G}_{x}=\left[\begin{array}{ccc}-1 & 0 & +1 \\ -2 & 0 & +2 \\ -1 & 0 & +1\end{array}\right] * \mathbf{A}$

Where $A$ is the origin image. Once you have $G_{x}$ and $G_{y}$ values, combine them this way:

$\mathrm{G}=\sqrt{\mathrm{G}_{x}^{2}+\mathrm{G}_{y}^{2}}$

to get the directional change and consequently the gray representation of the edge.

John Canny considered the mathematical problem of deriving an optimal smoothing filter given the criteria of detection, localization and minimizing multiple responses to a single edge [17]. He showed that the optimal filter given these assumptions is a sum of four exponential terms. He also showed that this filter can be well approximated by first-order derivatives of Gaussians. Canny also introduced the notion of non-maximum suppression, which means that given the presmoothing filters, edge points are defined as points where the gradient magnitude assumes a local maximum in the gradient direction. Looking for the zero crossing of the 2nd derivative along the gradient direction was first proposed by Haralick [18]. It took less than two decades to find a modern geometric variation meaning for that operator that links it to the Marr-Hildreth (zero crossing of the Laplacian) edge detector. That observation was presented by Ron Kimmel and Alfred Bruckstein[19].

Although his work was done in the early days of computer vision, Canny edge detector (including its variations) is still a state-of-the-art edge detector. Unless the preconditions are particularly suitable, it is hard to find an edge detector that performs significantly better than Canny edge detector.

The Canny-Deriche detector was derived from similar mathematical criteria as the Canny edge detector, although starting from a discrete viewpoint and then leading to a set of recursive filters for image smoothing instead of exponential filters or Gaussian filters.[20]

The differential edge detector described below can be seen as a reformulation of Canny's method from the viewpoint of differential invariants computed from a scale space representation leading to a number of advantages in terms of both theoretical analysis and sub-pixel implementation.

\section{Other first-order methods}

Different gradient operators can be applied to estimate image gradients from the input image or a smoothed version of it. The simplest approach is to use central differences:

$$
\begin{aligned}
& \mathrm{L}_{\mathrm{x}}(\mathrm{x}, \mathrm{y})=-1 / 2 \cdot \mathrm{L}_{\mathrm{x}}(\mathrm{x}-1, \mathrm{y})+0 . \mathrm{L}_{\mathrm{x}}(\mathrm{x}, \mathrm{y})+1 / 2 \cdot \mathrm{L}_{\mathrm{x}}(\mathrm{x}+1, \mathrm{y}) \\
& \mathrm{L}_{\mathrm{y}}(\mathrm{x}, \mathrm{y})=-1 / 2 . \mathrm{L}_{\mathrm{x}}(\mathrm{x}, \mathrm{y}-1)+0 . \mathrm{L}_{\mathrm{x}}(\mathrm{x}, \mathrm{y})+1 / 2 \cdot \mathrm{L}_{\mathrm{x}}(\mathrm{x}, \mathrm{y}+1)
\end{aligned}
$$

corresponding to the application of the following filter masks to the image data:

$L_{x}=\left[\begin{array}{lll}-1 / 2 & 0 & 1 / 2\end{array}\right] * L$ and $L_{y}=\left[\begin{array}{c}+1 / 2 \\ 0 \\ -1 / 2\end{array}\right] * L$. .

The well-known and earlier Sobel operator is based on the following filters:

$L_{x}=\left[\begin{array}{ccc}-1 & 0 & +1 \\ -2 & 0 & +2 \\ -1 & 0 & +1\end{array}\right] * L$ and $L_{y}=\left[\begin{array}{ccc}+1 & +2 & +1 \\ 0 & 0 & 0 \\ -1 & -2 & -1\end{array}\right] * L$.

Given such estimates of first- order derivatives, the gradient magnitude is then computed as:

$|\nabla \mathrm{L}|=\sqrt{\mathrm{L}_{\mathrm{x}}^{2}+\mathrm{L}_{y}^{2}} \cdots(7)$

while the gradient orientation can be estimated as:

$\theta=a \tan ^{2}\left(\mathrm{~L}_{\mathrm{x}}, \mathrm{L}_{\mathrm{y}}\right) \ldots$

\subsection{Color Edge Detection}

In 1977 Professor RamakantN. of USC published paper on color edge detection, in which he extended the Hueckel operator, developed 4 years previously, to color images. [21]

Nearly all of them still try to "extend" a greyscale edge detector to color images. While it seems intuitive to start with the solution to an easier problem in order to attack a more complex one, there is a drawback in this particular case. We perceive grey levels to be ordered; the fact that "medium grey" and "white" can be averaged together to produce "medium-light grey" does not bother anyone. However, if you take a red and green region and try to approximate it with a single color, you will run into problems immediately. Depending on your arrangement of colors, it could be yellow (in the spectrum), grey (CIE-Lab color space), 
yellowish-grey (RGB, HSV) or impossible (the opponent-colors theory). Even if we could agree on the "correct" color space, none of the colors mentioned is perceptually similar to red or green. This truth is the justification of the compass operator's use of color signatures. [21]

Most of the literature can be placed into three categories: output fusion methods, multi-dimensional gradient methods, and vector methods. Output fusion appears to be the most popular; the goal is to perform edge detection three times, once each for red, green, and blue (or whatever color space is being used), and then the output is fused to form one edge map, as shown by the following diagram:

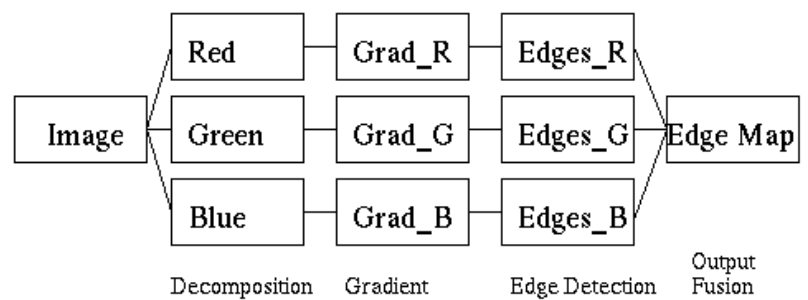

Multi-dimensional gradient methods short-circuit the process somewhat by combining the three gradients into one and detecting edges only once:

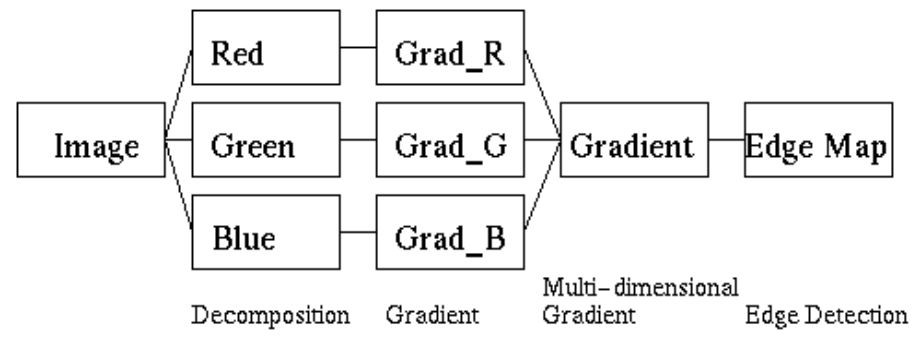

We find the decomposition of a multi-band image into a number of single-band images to be unpleasing. While it is true that humans perceive color in three dimensions, it is unlikely that edge detection or gradient computation takes place by projecting colors onto three separate axes.

\subsection{Conversion to and from RGB}

$\mathrm{Y}, \mathrm{Cb}$, and $\mathrm{Cr}$ are converted from $\mathrm{R}, \mathrm{G}$, and $\mathrm{B}$ as defined in CCIR Recommendation 601, but are normalized so as to occupy the full 256 levels of a 8-bit binary encoding. Moreprecisely: [22]

$$
\begin{aligned}
& \mathrm{Y}=256 * \mathrm{E}^{\prime} \mathrm{y} \\
& \mathrm{Cb}=256 *\left[\mathrm{E}^{\prime} \mathrm{Cb}\right]+128 \\
& \mathrm{Cr}=256 *\left[\mathrm{E}^{\prime} \mathrm{Cr}\right]+128 \ldots(9)
\end{aligned}
$$

where the $E^{\prime} y, E^{\prime} \mathrm{Cb}$ and $E^{\prime} \mathrm{Cb}$ are defined as in CCIR 601. Since values of $E^{\prime} y$ have arrange of 0 to 1.0 and those for $\mathrm{E}^{\prime} \mathrm{Cb}$ and $\mathrm{E}^{\prime} \mathrm{Cr}$ have a range of -0.5 to $+0.5, \mathrm{Y}, \mathrm{Cb}$, andCr must be clamped to 255 when they are maximum value.

\section{RGB to YCbCr Conversion[22]}

$\mathrm{YCbCr}$ (256 levels) can be computed directly from 8-bit RGB as follows:

$$
\begin{aligned}
& \mathrm{Y}=0.299 \mathrm{R}+0.587 \mathrm{G}+0.114 \mathrm{~B} \ldots(10 \mathrm{a}) \\
& \mathrm{Cb}=-0.1687 \mathrm{R}-0.3313 \mathrm{G}+0.5 \mathrm{~B}+128 \ldots(10 \mathrm{~b}) \\
& \mathrm{Cr}=0.5 \mathrm{R}-0.4187 \mathrm{G}-0.0813 \mathrm{~B}+128 \ldots(10 \mathrm{c})
\end{aligned}
$$

Note: Not all image file formats store image samples in the order $R_{0}, G_{0}, B_{0}, \ldots R_{n}, G_{n}, B_{n}$. Be sure to verify the sample order before converting an RGB file to JFIF.

\section{YCbCr to RGB Conversion[22]}

RGB can be computed directly from $\mathrm{YCbCr}$ (256 levels) as follows:

$$
\begin{aligned}
& \mathrm{R}=\mathrm{Y}+1.402(\mathrm{Cr}-128) \ldots(11 \mathrm{a}) \\
& \mathrm{G}=\mathrm{Y}-0.34414(\mathrm{Cb}-128)-0.71414(\mathrm{Cr}-128) \ldots(11 \mathrm{~b}) \\
& \mathrm{B}=\mathrm{Y}+1.772(\mathrm{Cb}-128) \ldots(11 \mathrm{c})
\end{aligned}
$$

\section{Samples Images and Algorithm}

Below, in figure (1), sample images of proposal method.And proposed scheme of Algorithm in figure (2). 

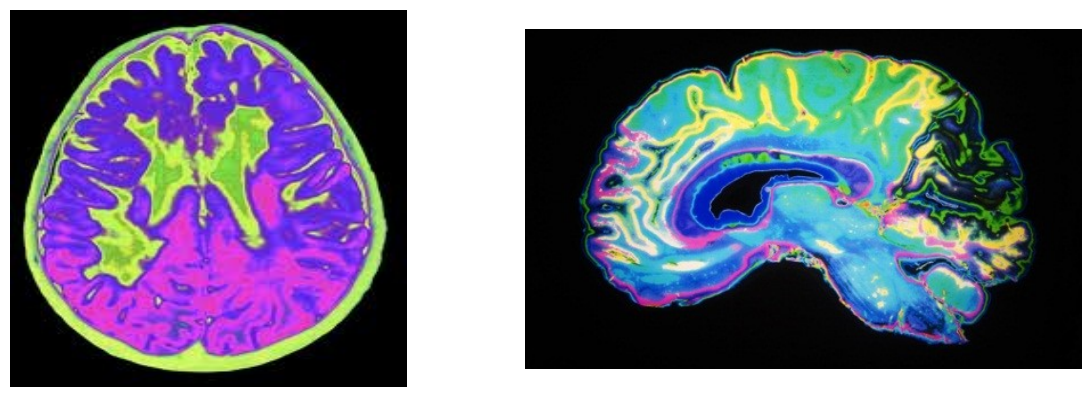

Figure (1)Images samples

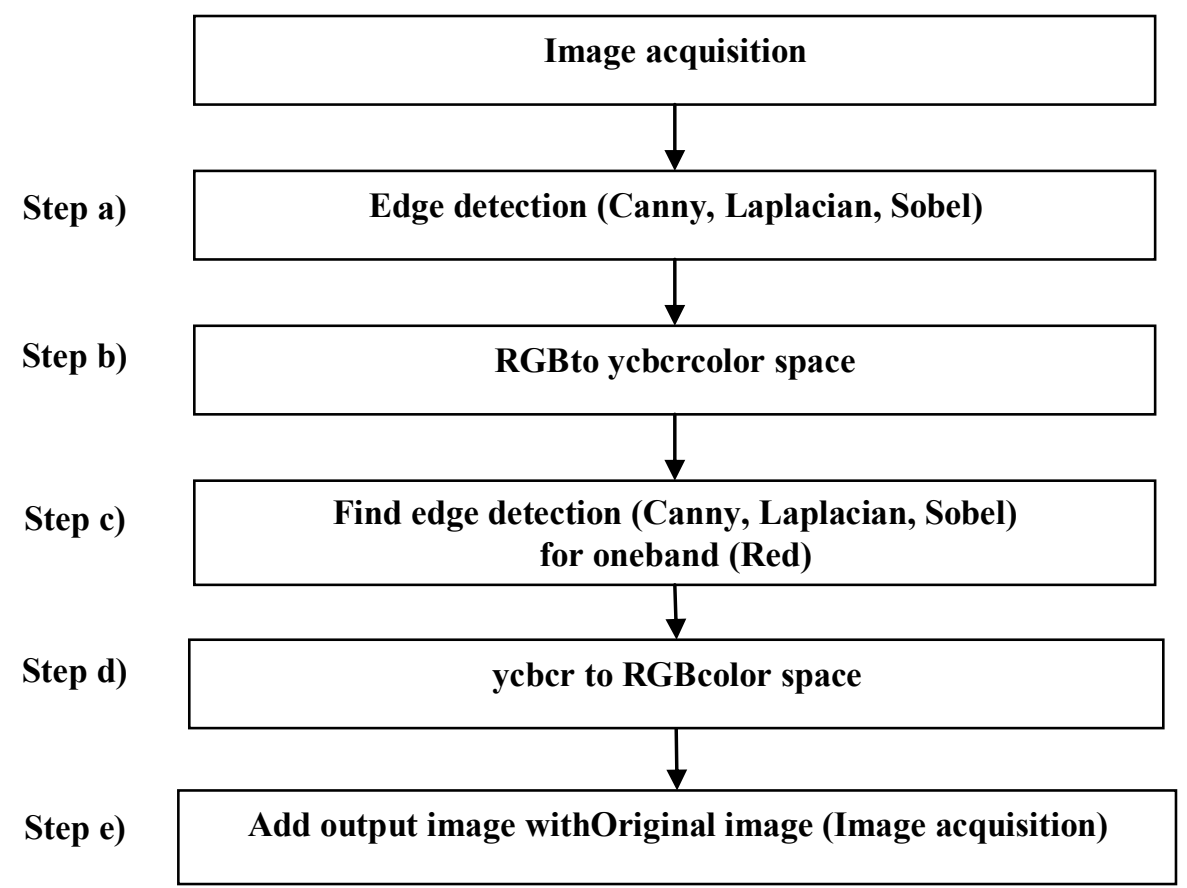

Figure (2) scheme proposed of Algorithm

IV. The Results:

Figure (3a, b) shows the results of application of proposal method on the images in figure (1) by Matlab2012a program.

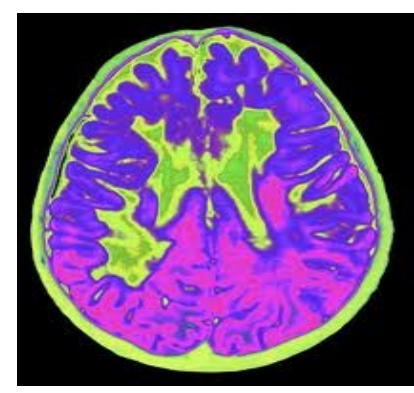

Original image no. 1 

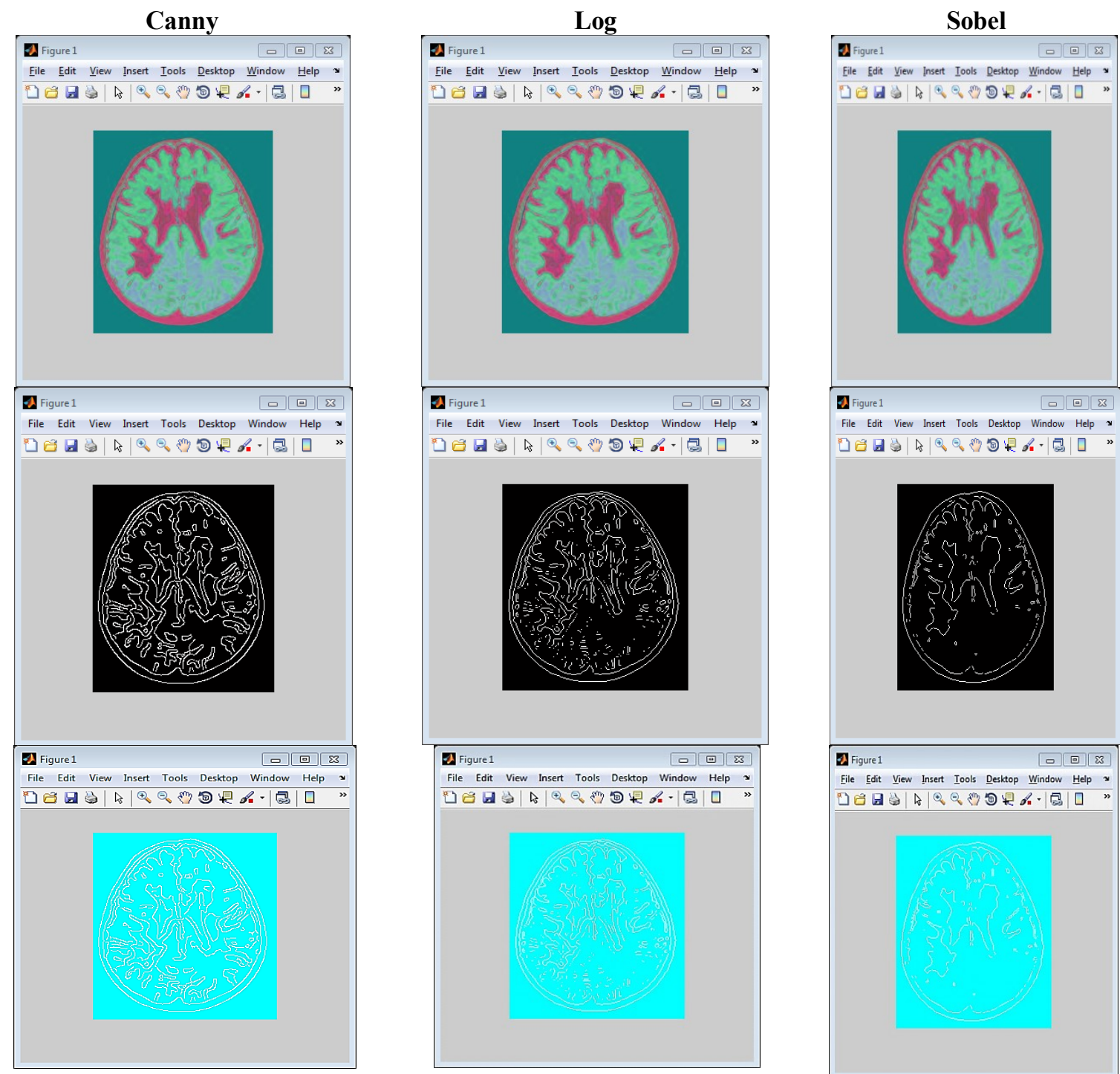

File Edit View Insert Tools Desktop Window Help y

b)

c)

File Edit View Insert Tools Desktop Window Help $~$

d)

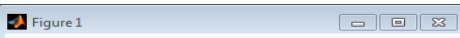

File Edit View Insert Tools Desktop Window Help $\simeq$

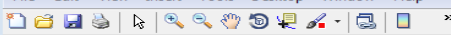

e)
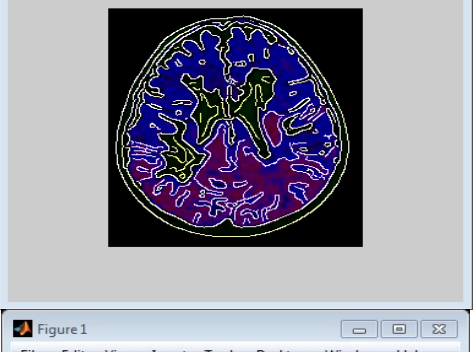
\begin{tabular}{l} 
File Edit View Insert Tools Desktop Window Help \\
$\square \square$ \\
\hline
\end{tabular}
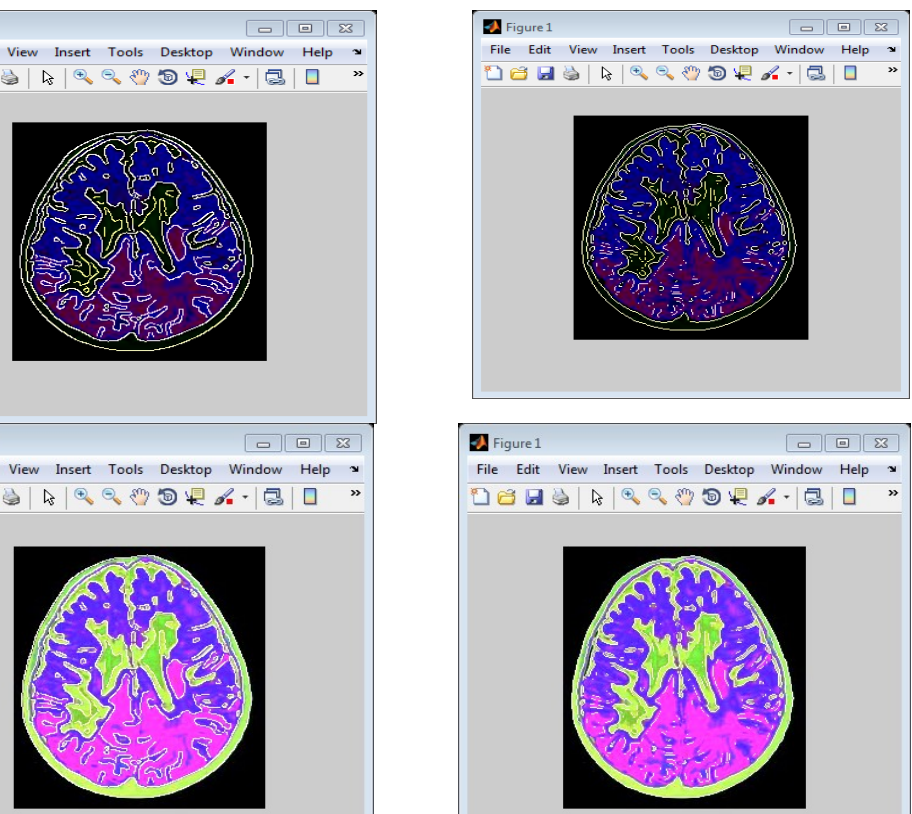

File Edit View Insert Tools Desktop Window Help =

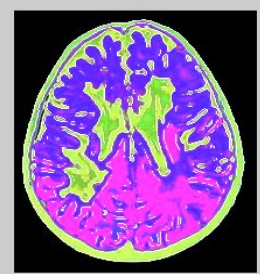

\begin{tabular}{lll}
\hline Figure 1 & \\
File Edit View Insert Iools Desktop Window Help & 83 \\
\hline
\end{tabular}

T)

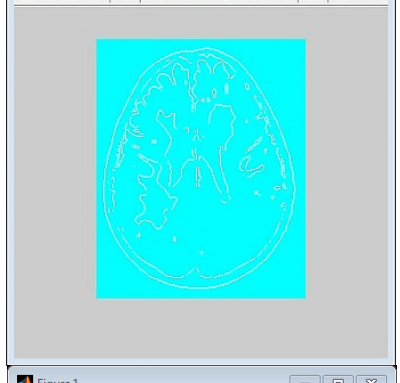

Q 20 Figure 1

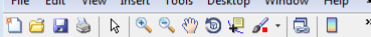
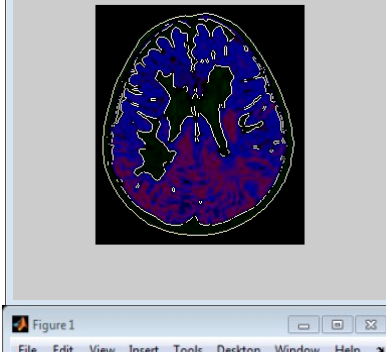

File Edit View Insert Tools Desktop Window Help $~ *$

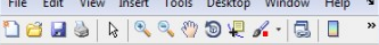

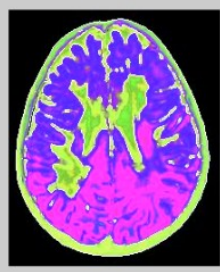

Figure (3a)Therustles of present workapplication on image no. 1 


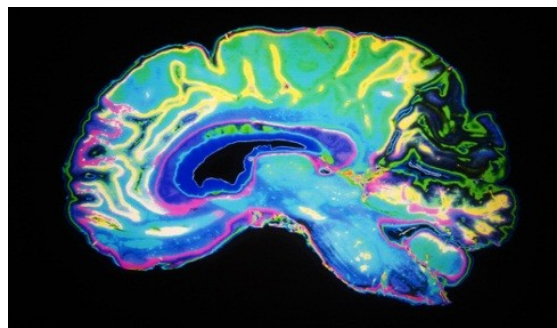

Original image no. 2
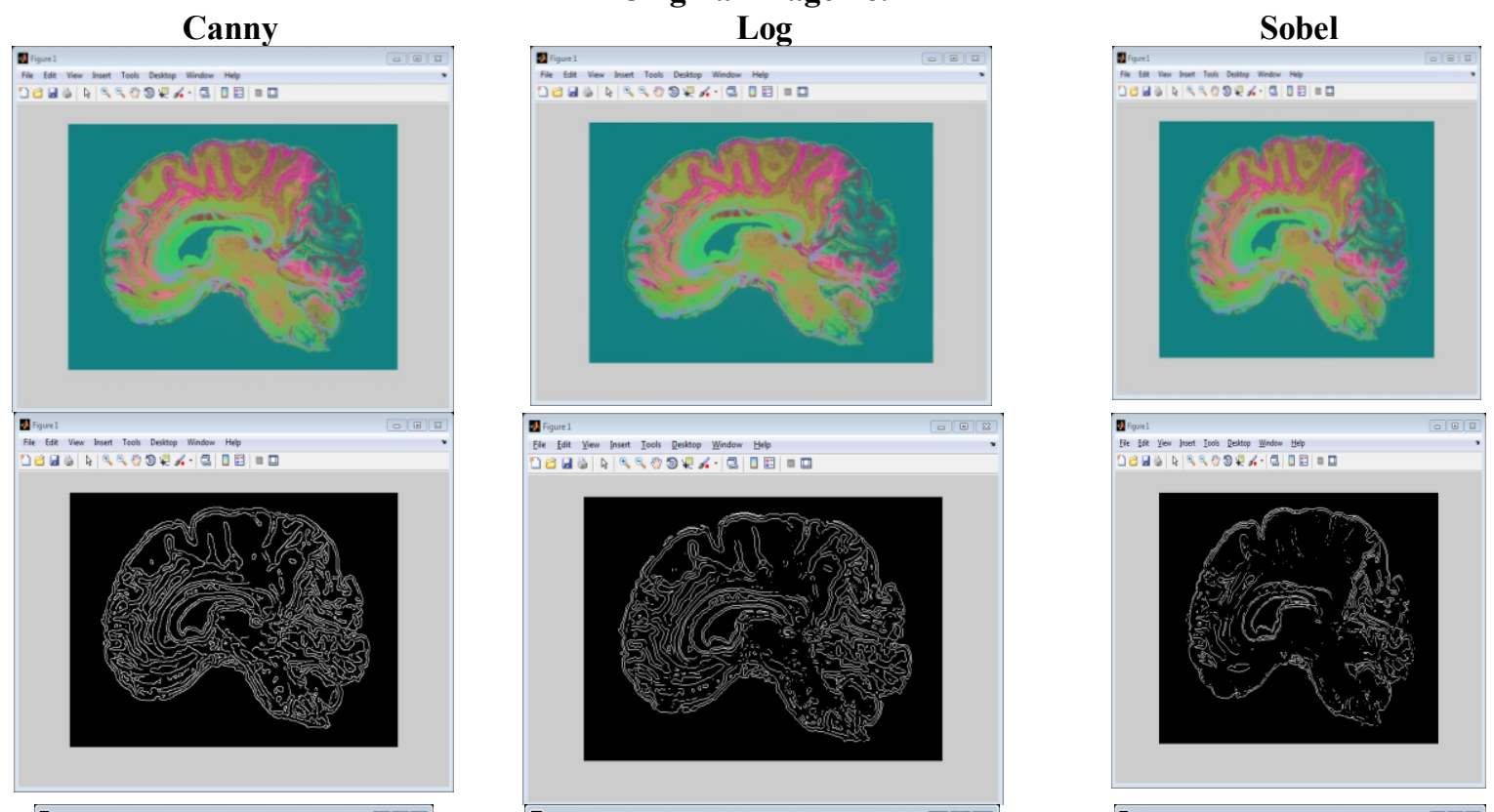

b)

c)
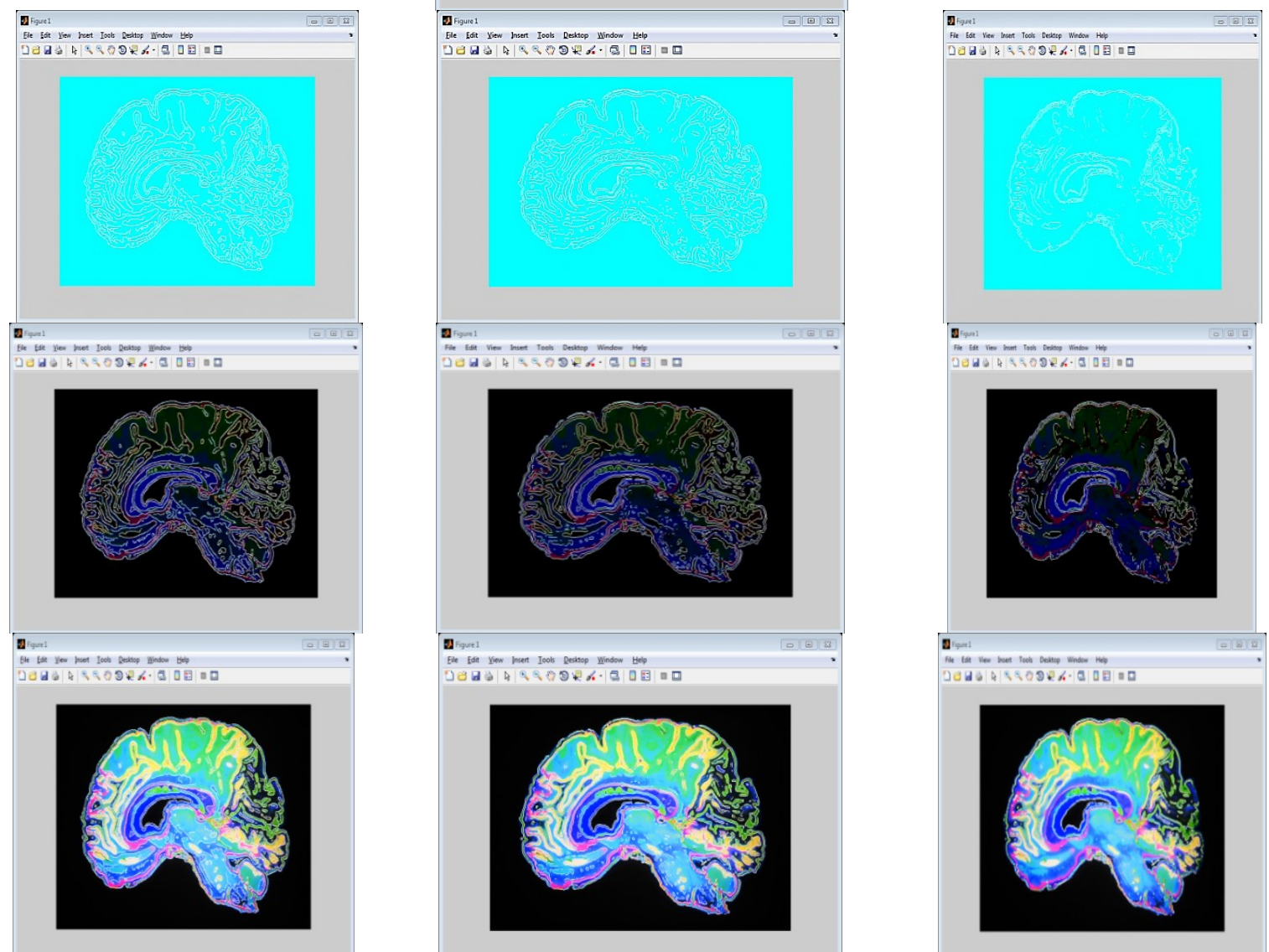

Figure (3b)The rustles of present work application on image no. 2 


\subsection{Conclusions:}

\section{Conclusions and Future works:}

1- The present method "add image with its edges" working to improve and increase the thickness of the edges of the color medical images (which is difficult to find their edges directly, such as grayscale images known filters), a special that captures a few due to poor quality image obtaining devices, whether or not the user experience. 2- This method helps in medical specialists see clearly on the edges of the resulting images of various types of medical images (MRI, CT, cat, Pat, ...) color and gray, with any size and any member of human's body.

\subsection{Future works:}

1. Apply other filters such as Prewitt and Roberts on the current image in the same method.

2. Application the current method pictures lawsuit in the field of remote sensing.

3. Application outdated video or unclear.

\section{References:}

[1] Mallat, S. G., "Multifrequency channel decompositions of image and wavelet models", IEEE Trans. Acoust. Speech Signal Process.vol. 37, no.12, pp. 2091-2110, 1989.

[2] Muna F. Al-Samaraie. Nedhal Abdul Majied Al Saiyd, "Medical Colored Image Enhancement Using Wavelet Transform Followed By Image Sharpening",vol. 6 No. 5, Ubiquitous Computing and Communication Journal, www.ubicc.org.

[3] Hajj, Hazern M.Nguyen, Truong Quang; Chin, Roland T. Multiscale edge detection for medical image enhancement, vol. 3, pp. 1115-1116, Conference Publications, IEEE, by ISVL,1996.

[4] Gudmundsson, M. El-Kwae, E.A.; Kabuka, M.R. Edge detection in medical images using a genetic algorithm, vol. 17, Issue: 3 , pp. 469-474, Journals \& Magazines, IEEE, by ISVL, 1998.

[5] Tarek A. Mahmoud, Stephen Marshall, Medical Image Enhancement Using Threshold Decomposition Driven Adaptive Morphological Filter, 16th European Signal Processing Conference (EUSIPCO 2008), Lausanne, Switzerland, August 25-29, 2008.

[6] Zhao Yu-qian, Gui Wei-hua, Chen Zhen-cheng, Tang Jing-tian, Li Ling-yun, Medical Images Edge Detection Based on Mathematical Morphology, Proceedings of the 2005 IEEE Engineering in Medicine and Biology 27th Annual Conference Shanghai, China, September 1-4, 2005.

[7] B. Lakshmi, G. Lingaiah, S. S. Madhavi, "An enhanced Approach for Medical edge Image enhancement using genetic algorithm", International Journal of Computer Science and technology y, ISSN : 0976-8491 (Online), ISSN: 2229-4333 (Print), IJCST, vol. 3, I 1, 2012,

[8] Gonzalez, R. C. and Woods, R. E. Digital Image Processing. ( $2^{\text {nd }}$ ed). Addison-Wesley Longman Publishing Co., Inc. 2001.

[9] He, Huiguang; Tian, Jie; Zhao, Mingchang; Xue, Jian; Lu, Ke, "3D Medical Imaging Computation and Analysis Platform", IEEE International Conference on Industrial, vol. 6 No. 5, p. 8, Technology ICIT, Dec. 2006.

[10] LathaParthiban; R. Subramanian, "Medical Image Denoising using X-lets", Annual India Conference, vol., Iss., pp. 1-6, Sept. 2006.

[11] D.Stark and W.Bradley Jr., Ed. Magnetic Resonance Imaging, St. Louis, MO: Mosby, 1992.

[12] Xu, D. and R. Wang, “An improved FoE model for image deblurring”. Int.J. Comput. Vis., 81: 167-171. DOI: 10.1007/s11263-0080155-3, 2009.

[13] Y. Watanabe, M. Dohke, T. Ishimori, Y. Amoh, K. Oda, A-Okumura, K. Mitsudo, and Y. Dodo, High-resolution MRCholangiopancreatography, Critical Rev Diagno Imaging, vol. 39, pp. 111-258, 1998.

[14] Michael J. Greaney, Douglas C. Hoffman, David F. Garway-Heath, MamdouhNakla, Anne L. Colemna, and Caprioli, "Comparison of Optic Nerve Imaging Methods to Distinguish Normal Eyes from Those with Glaucoma”, IOVS, vol. 43, No. 1, pp. 140- 145, 2002

[15] Huiqi Li, and OpasChutatape, "Automated Feature Extraction in Color Retinal Images by a Model Based Approach", IEEE Transaction on Biomedical Engineering, vol. 51, no. 2, pp. 246-254, 2004.

[16] Yan, X., L. Kai-Yang, Y. Xuan-Dong, W. X. Fang and Z. X. Lin et al,“"The application of the edge sharpening operator to the breast near infrared”. Wuhan Univ. J. Nat. Sci., 2002, 7: 421425. DOI: 10.1007/BF02828241.

[17] Charles Poynton, "Digital Video and HDTV”, Chapter 24, pp. 291-292, Morgan Kaufmann, 2003.

[18] Alparone, L., L. Wald, J. Chanussot, C. Thomass and P. Gamba et al. Comparison of pan sharpening algorithms: Outcome of the 2006 GRS-S data-fusion contest. IEEE Trans. Geosci. Remote Sens., 45: 3012-3021. DOI: 10.1109/TGRS.2007.904923, 2007.

[19] Welsh, T., M. Ashikhmin and K. Mueller, Transferring color to greyscale images. ACM Trans. Graph., 21: 277-280. DOI: $10.1145 / 566570.566576,2002$.

[20] Zhang X, Wandell B A, "A spatial extension to CIELAB for digital color image reproduction," Soc. for Info Disp.Symp. Tech Digest; 27:731-734, 1996.

[21] Mark A. Ruzon, “A Short History of Color Edge Detection”.www.ai.stanford.edu

[22] Eric Hamilton, "JPEG File Interchange Format", Version 1.02, 1992. 